Clinical Scholarship

\title{
The Influence of Caregiver Mastery on Depressive Symptoms
}

Paula R. Sherwood, Barbara A. Given, Charles W. Given, Rachel F. Schiffman, Daniel L. Murman, Alexander von Eye, Mary Lovely, Lisa R. Rogers, Sandy Remer

\begin{abstract}
Purpose: The purpose of this study was to explore how the relationship between care recipients' problem behaviors and caregivers' depressive symptoms varies as a function of caregiver mastery, controlling for the effects of caregiver age, gender, and relationship to the care recipient in caregivers of people with primary malignant brain tumor (PMBT).

Design: A cross-sectional design was used to gather data via telephone interviews from 95 caregivers of people with primary malignant brain tumor, recruited from 2003 to 2004 from a brain tumor treatment center, two national support groups, and a statewide cancer registry.

Methods: Measures for the study included the Neuropsychiatric Inventory-Questionnaire, Caregiver Mastery, and the Center for Epidemiologic Studies-Depression. A stepwise regression procedure was used to evaluate potential moderating and mediating relationships.

Findings: Data did not indicate that caregiver mastery was a moderating variable. The anal$y$ sis showed caregiver mastery as a partial mediator, with both a direct effect of care recipients' problem behaviors on caregivers' depressive symptoms and an indirect effect through caregiver mastery. Concerning the indirect effect, care recipients' problem behaviors were related to lower levels of caregiver mastery, which in turn were related to more depressive symptoms in caregivers.

Conclusions: Findings showed a link between care recipients' problem behaviors and caregivers' depressive symptoms, a relationship that has not been well established in oncology. This association indicates one mechanism through which problem behaviors in the care recipient might lead to caregiver depressive symptoms.
\end{abstract}

Journal of Nursing Scholarship, 2007; 39:3, 249-255. C2007 Sigma Theta Tau International.

[Key words: caregiver, oncology, mastery, mediating, brain tumor]

$\mathrm{M}$ uch caregiver research has been devoted to describing changes in the physical and emotional health of family members who provide care to someone with a chronic illness. Outcomes such as depressive symptoms, perceived burden, anxiety, and changes in immune function have all been linked to providing care (Kiecolt-Glaser et al., 2003; Kozachik et al., 2001; Pinquart $\&$ Sörensen, 2004). Investigators have also identified specific care demands, such as care recipients' behavioral and cognitive problems that are strongly associated with changes in caregivers' health (Pinquart \& Sörensen, 2004). Not all caregivers respond similarly to the challenges of providing care. For example, in the presence of similar stressors, some caregivers develop high levels of depressive symptoms, but others do not (Mahoney, Regan, Katona, \& Livingston, 2005).

Caregiver characteristics such as age, gender, and relationship to the care recipient have been shown to help identify caregivers at risk for negative consequences as a result of providing care (Bookwala \& Schulz, 2000;
Paula R. Sherwood, RN, PhD, CNRN, Alpha Psi, Assistant Professor, University of Pittsburgh School of Nursing, Pittsburgh, PA; Barbara A. Given, RN, PhD, FAAN, Alpha Psi, University Distinguished Professor; College of Nursing, Michigan State University, East Lansing, MI; Charles W. Given, PhD, Professor; College of Medicine, Michigan State University, East Lansing, Ml; Rachel F. Schiffman, RN, PhD, FAAN, Alpha Psi and Eta Nu, Professor and Associate Dean for Research; College of Nursing, University of Wisconsin-Milwaukee, WI; Daniel L. Murman, MD, MS, Associate Professor; Department of Neurological Sciences, University of Nebraska Medical Center, Omaha, NE; Alexander von Eye, PhD, Professor; Department of Psychology, East Lansing, Ml; Mary Lovely, RN, PhD, Medical Information Specialist, Supported by the Gullikson Foundation; National Brain Tumor Foundation, CA; Lisa R. Rogers, DO, Professor of Neurology, University of Michigan, Ann Arbor, Ml; Sandy Remer, RN, BS, OCN ${ }^{\circledR}$, CCRP, Neuro-Oncology Coordinator; Josephine Ford Cancer Center, Henry Ford Hospital, Detroit, MI. Support for the project was provided by the National Institute of Nursing Research (F31NR8069); Oncology Nursing Society, the American Brain Tumor Association, American Cancer Society, Sigma Theta Tau International, Mary Margaret Walther Cancer Institute, and Michigan State University College of Nursing. Correspondence to Dr. Sherwood, University of Pittsburgh, School of Nursing, 336 Victoria Bldg, 3500 Victoria St.; Pittsburgh, PA 15261. E-mail: prs11@pitt.edu Accepted for publication April 5, 2007. 
Mahoney et al., 2005; Nijboer, Tempelaar, Triemstra, van den Bos, \& Sanderman 2001; Northouse, Mood, Templin, Mellon, \& George, 2000). More recently, nonsociodemographic caregiver characteristics have been proposed as variables that might influence the relationship between care demands and caregiver outcomes. One such characteristic is caregiver mastery, which can be defined as caregivers' feelings of being in control of the care situation, and which appears to influence the relationship between care recipients' problem behaviors (such as hallucinations and agitation) and caregivers' level of depressive symptoms; however, whether caregiver mastery moderates or mediates this relationship is not well established.

From a healthcare system perspective, understanding the moderating or mediating role of caregiver characteristics is a necessary component of efficiently implementing caregiver interventions. Understanding the mechanisms through which variables in the care situation affect caregiver outcomes allows practitioners to more appropriately monitor caregivers at risk for distress, more precisely target interventions to the variables leading to distress, and more accurately evaluate outcomes. The purpose of this study was to explore whether caregiver mastery moderates or mediates the relationship between care demands and caregiver outcomes. Specifically, the study was designed to explore how the relationship between care recipients' problem behaviors and caregivers' depressive symptoms varies as a function of caregiver mastery, controlling for the effects of caregiver age, gender, and relationship to the care recipient, in caregivers of people with a primary malignant brain tumor (PMBT).

\section{Background}

A global sense of mastery has been defined as the amount of control that people feel over the forces impinging upon them (Pearlin \& Schooler, 1978). The concept of mastery was originally developed to describe a person's general sense of control over their lives, referred to in this paper as "global mastery." Researchers in the area of family caregiving have applied the concept of mastery to the care situation. "Caregiver mastery" delineates caregivers' perceptions of their sense of worth as caregiver and how they perceive their ability to meet the demands of providing care (Gaugler et al., 2005; Gitlin et al., 2003). People with high levels of caregiver mastery report that they are able to face the challenges of providing care, feel in control of the care situation, and are able to use problem-solving skills to meet care demands, which may result in fewer depressive symptoms (Bookwala \& Schulz, 1998).

Research has shown that caregivers' sense of mastery is important in determining the degree to which providing care will affect their susceptibility to developing depressive symptoms. Bookwala and Schulz (1998) examined data from 378 caregivers of people 65 years of age or older with various chronic medical conditions and found that low levels of global mastery predicted higher levels of caregiver strain and depressive symptoms. These findings were supported by $\mathrm{Li}$ et al. (1999) in their study of daughter-caregivers of people over the age of 60 with various medical conditions.

However, Bookwala et al.'s (1998) and Li et al.'s (1999) studies differed regarding the way in which caregiver mastery influenced caregiver outcomes when problem behaviors were present in care recipients. Bookwala and Schulz found that when caregiver mastery was included as a predictor of caregiver outcomes, the amount of variance in caregivers' depressive symptoms that was accounted for by care recipients' behavior problems decreased by approximately $8 \%$. These data indicated a moderating role of caregiver mastery, i.e., that the strength of the relationship between care recipients' behavior problems and caregivers' depressive symptoms is dependent upon caregivers' level of mastery. The results from Li et al.'s study, on the other hand, indicated that caregiver mastery was a partial mediator (having both an indirect and direct effect) of the relationship between care recipients' problem behaviors and caregivers' depressive symptoms. Li et al. found a direct relationship between care recipients' problem behaviors and caregivers' depressive symptoms, but also reported that problem behaviors led to lower levels of caregiver mastery, which in turn, led to higher levels of caregivers' depressive symptoms (an indirect relationship). Skaff, Pearlin, and Mullan (1996) reported that in caregivers of people with Alzheimer's disease, problem behaviors in the care recipient were significant predictors of changes in caregiver mastery over time, which may also support a mediating effect of caregiver mastery.

In the area of oncology, Nijboer and colleagues (2001) reported that levels of global mastery predicted changes in depressive symptoms over time in caregivers of people with newly diagnosed colorectal cancer. In addition, the authors found that caregiver mastery appeared to moderate the relationship between care recipients' depressive symptoms and caregivers' depressive symptoms: when caregivers had high levels of caregiver mastery, they had lower levels of depressive symptoms as a result of care recipients' depressive symptoms. Gaugler and colleagues (2005) reported that caregivers of people with various types of cancer who reported higher levels of caregiver mastery had less emotional distress, but they did not explore the moderating or mediating role of caregiver mastery in this sample.

Although Nijboer et al. (2001) and Gaugler et al. (2005) have provided more data regarding the influence of caregiver mastery in caregiver and care recipient relationships in oncology, no studies in oncology to date have been reported on the role of caregiver mastery in the presence of problem behaviors in care recipients. Problem behaviors are not common among all types of cancer, but when present (such as in people with a primary brain tumor or those with metastatic lesions to the brain), problem behaviors can be a great source of distress for the caregiver. Problem behaviors such as agitation, hallucinations, and delusions may have multiple etiologies. They have strong effects on the distress of caregivers as a result of providing care. Problem behaviors can hinder a care recipients' ability to perform ADLs and 
IADLs, increasing the need for assistance from caregivers (Ory, Hoffman, Yee, Tennstedt, \& Schulz, 1999). Problem behaviors of care recipients might lead caregivers to believe that they are providing care for strangers (Salander, 1996), which can increase the distress they feel as a result of providing care.

Further, problem behaviors might affect the availability of secondary carers. Caregivers have reported that other family members and friends are less willing to be involved in sharing care responsibilities when the care recipient has behavioral problems (Sherwood, Given, Doorenbos, \& Given, 2004) than when functional problems alone are present. Our previous work showed a strong relationship between care recipients' problem behaviors and caregivers' depressive symptoms (Sherwood et al., 2006); this analysis was done to determine characteristics that might influence that relationship.

Many of the previously mentioned studies were focused on the influence of global mastery, rather than caregiver mastery (or mastery specific to the care situation), in the relationship between care recipients' problem behaviors and caregivers' depressive symptoms. Global mastery could be a personality characteristic or trait that might not be amenable to intervention. Using measures of global mastery in caregivers might result only in identifying those in need of intervention. Caregiver mastery, on the other hand, can be viewed as situation-specific or a "state" characteristic, one which directly results from feelings of control over the care situation. By considering caregiver mastery in this way to identify caregivers at risk for depressive symptoms, health care practitioners might be able to improve caregivers' sense of mastery, thus lowering the risk for emotional distress. For this reason, we chose to evaluate the role of caregiver, rather than global, mastery in the care situation.

Caregiver mastery might either moderate or mediate the relationship between care recipients' problem behaviors and caregivers' depressive symptoms. According to the models proposed by Baron and Kenny (1986; Kenny, 2005), if caregiver mastery is a moderating variable, then the caregiver's level of mastery will change the way in which problem behaviors in the care recipient affect caregivers' depressive symptoms. Caregivers of people with numerous problem behaviors might not display depressive symptoms if the caregiver's level of mastery is high. In this case, caregiver mastery should be used to monitor caregivers of people with problem behaviors. Clinicians who wish to intervene to improve caregiver depressive symptoms, then, would target caregivers with low levels of caregiver mastery and would work with caregivers to increase their levels of caregiver mastery in the presence of problem behaviors in care recipients.

If caregivers mastery is a mediating variable, the caregivers' level of mastery will be dependent upon care recipients' problem behaviors. Increasing problem behaviors in care recipients will cause lower levels of caregiver mastery, which will in turn lead to higher levels of depressive symptoms. In this case, clinicians would assess the number and severity of problem behaviors in the care recipient in a risk assessment for those caregivers at risk for depressive symptoms. Clinicians would then focus on assisting caregivers in managing problem behaviors to improve levels of caregiver mastery before low levels of caregiver mastery lead to high levels of depressive symptoms. Thus, the purpose of this study was to apply Baron and Kenny's (1986; Kenny, 2005) framework to determine whether caregiver mastery mediated or moderated the relationship between care recipients' problem behaviors and caregivers' depressive symptoms in the situation of primary malignant brain tumors.

\section{Methods}

\section{Setting and Sample}

Institutional review board approval was obtained from the principal investigator's institution, in addition to the governing bodies of each of the recruitment sites. Caregivers for the study were recruited from five organizations: two metropolitan brain tumor treatment centers, two national brain tumor support organizations, and one statewide cancer registry. A caregiver was defined as someone who provided assistance to the care recipient (e.g., emotional assistance, physical assistance, monetary assistance). Criteria for caregiver eligibility were: (a) being over 21 years of age, (b) caring for someone with a primary malignant brain tumor who was over 21 years of age, (c) being fluent in English, and (d) having regular and reliable access to a telephone. Recruitment strategies and sociodemographic characteristics of the sample have been described elsewhere (Sherwood et al., 2006). Caregivers were either approached for consent in person (if geographically possible) or consent forms were mailed to caregivers, who then called to express interest in participating.

A total of 95 participants were recruited for the study. The majority of participants (age range 25-76 years, $M=51$, $S D=12)$ were women $(74 \%, n=70)$, Caucasian $(94 \%$, $n=89)$, and spouses $(74 \%, n=70)$ of care recipients. Most caregivers had provided care for less than 3 years $(77 \%$, $n=73$; range 1 to 216 months, $M=31, S D=36$, median=18 ). The most common tumor type for care recipients (age range $21-78$ years, $M=48, S D=14$ ) was glioblastoma multiforme (GBM; 44\%, $n=42$ ).

\section{Data Collection}

After consent was obtained, the caregiver underwent a 45- to 90 -minute telephone interview by a trained interviewer $(M=72$ minutes), during which measures to assess the following variables were administered: care recipient problem behaviors, caregiver mastery, and caregiver depressive symptoms. Telephone interviews were used to obtain data because caregivers were recruited from across the United States.

The care recipient's problem behaviors were measured with the Neuropsychiatric Inventory-Questionnaire (NPIQ; Kaufer et al., 2000). The NPI-Q $(\alpha=.78)$ is a 12 -item 
measure in which the caregiver is asked to indicate the presence of 12 abnormal care recipient behaviors (e.g., delusions and hallucinations). Each behavior is rated as either present or absent; scoring for the NPI-Q consisted of summing individual items to generate a total score, with higher scores indicating more abnormal behaviors (possible range 0 to12). Validity for the NPI-Q has been established in people with neurodegenerative disorders and in older hospitalized patients on acute care units (Kaufer et al.; O'Hara et al., 2002).

Caregiver mastery was assessed with the seven-item mastery scale which was adapted from Pearlin and Schooler's (1978) scale to indicate mastery over the care situation $(\alpha=.73)$. Caregivers were asked to use a 5 point Likert-type scale to indicate their perceptions of how certain they were about what to do in providing care, how they perceived themselves as able to handle most of the problems they faced in the care situation, and how well they believed that they were mastering most of the challenges in caregiving. Scoring for the caregiver mastery scale was done by summing item scores to generate a total score; higher scores indicated higher levels of caregiver mastery (possible range 7 to 35$)$.

Caregivers' depressive symptoms were assessed with the Center for Epidemiologic Studies-Depression scale (CES-D; Radloff, 1977). The CES-D $(\alpha=.85)$ has been shown to be a valid measure of depressive symptoms in adults (Andresen, Malmgren, Carter, \& Patrick, 1994). It is a 20 -item scale to assess the respondent's current level of depressive symptoms on a 4-point Likert-type scale. Scoring the CES-D consisted of summing individual items to produce a total score; higher numbers indicated the presence of more depressive symptoms (possible range 0 to 60 ).

Potential confounding variables considered for inclusion in each model, based on literature in the field of dementia and oncology caregiving, were caregiver age, gender, and relationship to the care recipient (Kozachik et al., 2001; Pinquart \& Sörensen, Pinquart, \& Duberstein, 2002). Caregiver gender and relationship to the care recipient (spouse and nonspouse) were dummy coded with female and spouse as referents. Relationship to the care recipient did not affect any of the proposed relationships and was deleted from the final analyses.

\section{Statistical Analysis}

Analyses to determine the moderating or mediating effects of caregiver mastery on the relationship between care recipients' problem behaviors and caregivers' depressive symptoms were based on the principles proposed by Baron and Kenny (1986; Kenny, 2005). To test moderation, two stepwise regressions were performed with caregiver depressive symptoms as the dependent variable. In the first regression model, care recipient problem behaviors, caregiver mastery, caregiver age, and caregiver gender were entered into the model. In the second regression model, the interaction term generated by the product of problem behaviors and caregiver mastery was entered. To test a potential me- diating effect, three regression equations were performed, controlling for caregiver age and gender: (a) the effect of care recipients' problem behaviors on caregiver mastery, (b) the effect of care recipients' problem behaviors on caregiver depressive symptoms, and (c) the effect of care recipients' problem behaviors and caregiver mastery on caregiver depressive symptoms.

\section{Findings}

Results from the moderation analyses are shown in Table 1. Preliminary analysis showed a large amount of collinearity between problem behaviors and caregiver mastery, and these two values were centered on the mean before regression analysis. In the first stepwise regression, both care recipients' problem behaviors $(p<.01)$ and caregiver mastery $(p<.01)$ predicted caregiver depressive symptoms. In the second stepwise regression, care recipients' problem behaviors and caregiver mastery were still predictive of caregiver depressive symptoms, but the interaction between the two was not significant $(p=.11)$ and did not indicate that caregiver mastery was a moderating variable.

To test caregiver mastery as a mediating variable, three regression equations were completed and their results compared (see Table 2 and Figure). Data from the first equation showed that controlling for caregiver age and gender, care

\begin{tabular}{|c|c|c|c|}
\hline \multicolumn{4}{|c|}{$\begin{array}{l}\text { First stepwise regression. The effect of care recipients' } \\
\text { problem behaviors,caregiver age, caregiver gender, and } \\
\text { caregiver mastery on caregiver depressive symptoms; } \\
\qquad R^{2}=.48(S E=6.86)\end{array}$} \\
\hline Variable & & $S E$ & $t$ \\
\hline Constant & & 3.45 & $18.37^{*}$ \\
\hline Care recipients' & & .27 & $4.02^{*}$ \\
\hline Caregiver gender & & 1.80 & $-3.75^{*}$ \\
\hline Caregiver age & & .07 & $-2.67^{*}$ \\
\hline Caregiver mastery & & .19 & $-4.07^{*}$ \\
\hline \multicolumn{4}{|c|}{$\begin{array}{l}\text { Second stepwise regression. The effect of care recipients' problem behaviors, } \\
\text { caregiver age, caregiver gender, caregiver mastery, and the interaction of } \\
\text { problem behaviors x mastery on caregiver depressive symptoms; } \\
\qquad R^{2}=.48(S E=6.86)\end{array}$} \\
\hline Variable & Beta & $S E$ & $t$ \\
\hline Constant & 26.36 & 3.44 & $7.66^{*}$ \\
\hline Care recipients' problem behaviors & 1.11 & .27 & $4.08^{*}$ \\
\hline Caregiver gender & -6.94 & 1.79 & $-3.89 *$ \\
\hline Caregiver age & -.18 & .07 & $-2.77^{*}$ \\
\hline Caregiver mastery & -.90 & .20 & $-4.41^{*}$ \\
\hline Problem behaviors X Mastery & .09 & .05 & 1.60 \\
\hline
\end{tabular}




\begin{tabular}{|c|c|c|c|}
\hline \multicolumn{4}{|c|}{$\begin{array}{l}\text { Equation 1. Effect of care recipients' problem behaviors on caregiver } \\
\qquad \text { mastery; } R^{2}=.09(S E=4.12)\end{array}$} \\
\hline Variable & Beta & $S E$ & $t$ \\
\hline $\begin{array}{l}\text { Care recipients' } \\
\text { problem behaviors }\end{array}$ & -.41 & 0.15 & $-2.65^{*}$ \\
\hline Caregiver gender & -1.24 & 1.03 & -1.21 \\
\hline Caregiver age & .0005 & .04 & .01 \\
\hline
\end{tabular}

Equation 2. Effect of care recipients' problem behaviors on caregiver depressive symptoms; $R^{2}=.35(S E=7.58)$

\begin{tabular}{lrrr} 
Variable & Beta & $S E$ & $t$ \\
\hline Care recipients' & 1.42 & .29 & $4.94^{*}$ \\
$\quad$ problem behaviors & & & \\
Caregiver gender & -5.95 & 1.96 & $-3.04^{*}$ \\
Caregiver age & -.18 & .07 & $-2.49^{*}$ \\
\hline
\end{tabular}

Equation 3. Effect of care recipients' problem behaviors and caregiver mastery on caregiver depressive symptoms; $R^{2}=.47(S E=6.93)$

\begin{tabular}{lccc} 
Variable & Beta & SE & $t$ \\
\hline Care recipients' & 1.10 & .27 & $4.02^{*}$ \\
$\quad$ problem behaviors & & & \\
Caregiver mastery & -.76 & .19 & $-4.07^{*}$ \\
Caregiver gender & -6.75 & 1.80 & $-3.75^{*}$ \\
Caregiver age & -.18 & .07 & $-2.67^{*}$ \\
\hline
\end{tabular}

$* p<.05$

Note. Confounding variables: Caregiver age*, caregiver gender*; all values significant at $p<.01 ; R^{2}=.47$

recipients' problem behaviors predicted $(p=.01)$ caregiver mastery, caregivers of people with higher numbers of problem behaviors reported lower levels of caregiver mastery.

The second equation showed that care recipients' problem behaviors $(p<.01)$, along with caregiver age $(p=.02)$ and caregiver gender $(p<.01)$, predicted caregiver depressive symptoms. Regarding age and gender, younger caregivers and female caregivers had higher levels of depressive symptoms. Women had a mean depressive symptom score of $16.13(S D=9.20)$, compared to a mean of $11.26(S D=7.28)$ for men, a significant difference $(t=2.31, p=.02)$.

The final test for mediation was to enter both the hypothesized independent and mediating variables into a regression equation and evaluate: (a) whether the mediating variable was a significant predictor of the outcome variable, and (b) whether the strength of the relationship between the independent and the outcome variable decreased when the mediating variable was included (Bennett, 2000). When both care recipients' problem behaviors and caregiver mastery were included as predictors of caregiver depressive symptoms, controlling for caregiver age and gender, the model accounted for $47 \%$ of the variance, and both care

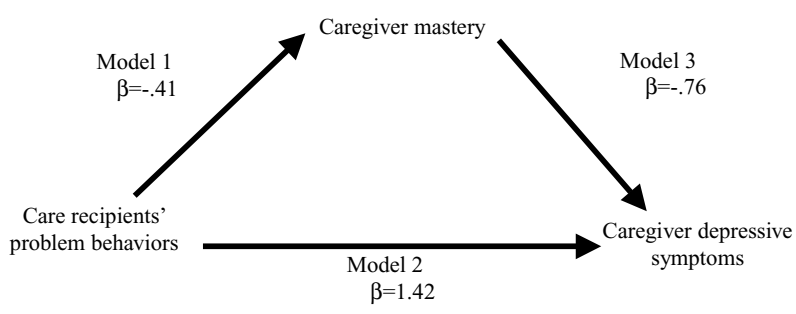

Note. Confounding variables: caregiver age*, caregiver gender*; all values significant at $p<.01 ; R^{2}=.47$.

Figure. Model of the partial mediation of caregiver mastery in the relationship between care recipients' problem behaviors and caregivers' depressive symptoms.

recipients' problem behaviors $(p<.01)$ and caregivers' mastery $(p<.01)$ were significant predictors of depressive symptoms, satisfying the first criterion. Concerning the second criterion, the $t$-value representing the relationship between care recipients' problem behaviors and caregivers' depressive symptoms decreased from 4.94 when caregiver mastery was not included (see Table 2) to 4.02 when caregiver mastery was included. Sobel's test to determine whether this change was statistically significant was performed, and the result $(p=.03)$ indicated a significant difference. The effect of care recipients' problem behaviors on caregivers' depressive symptoms, however, did not completely disappear when caregiver mastery was entered into the model, indicating that a partial mediation had occurred (see Figure). Partial mediation can show that care recipients' problem behaviors directly affected caregivers' depressive symptoms, but also indirectly affected depressive symptoms by lowering levels of caregiver mastery.

\section{Discussion}

Data from caregivers of people with PMBTs were examined to explore how caregiver mastery influenced the way in which care recipients' problem behaviors predicted caregivers' depressive symptoms. The findings were not consistent with Nijober et al.'s (2001) findings of a moderating relationship; i.e., that the effect of care recipients' problem behaviors on caregivers' depressive symptoms varied according to level of caregiver mastery. One hypothesis might be that this study differed from Nijboer et al's work as a result of the sample. Nijboer et al. evaluated caregivers of people with colorectal cancer, care recipients who were more likely to have physical, rather than neurologic, symptoms. The effects of caregiver mastery might vary when problem behaviors, rather than physical symptoms, are present. The inability to identify a moderating relationship may also have resulted from measurement error. The reliability of the instruments used to assess problem behaviors and caregiver mastery may not have been high enough to overcome issues related to measurement error. Future analysis with more objective measures of care recipient problem behaviors and a 
measure of caregiver mastery with higher reliability should be done to further investigate this relationship.

Instead, the analysis showed a partial mediation: both a direct effect of care recipients' problem behaviors on caregivers' depressive symptoms and an indirect effect through caregiver mastery. Concerning the indirect effect, care recipients' problem behaviors led to lower levels of caregiver mastery, which in turn led to higher numbers of depressive symptoms in caregivers, providing insight into one mechanism through which problem behaviors in the care recipient might lead to caregiver depressive symptoms. Results indicated that, as the number of problem behaviors in care recipients increased, caregivers began to feel less in control of the care situation. Possibly, caregivers feel confident handling one or two problem behaviors such as depression and irritability. However, when care recipients' problem behaviors increase, caregivers have fewer resources with which to manage those behaviors, leading to feelings of being out of control and not knowing how to handle care demands (i.e., they have lower levels of caregiver mastery).

Clinicians might therefore use the number of problem behaviors in the care recipient to identify caregivers at risk for decreased levels of caregiver mastery. Clinicians interested in reducing caregivers' depressive symptoms, then, could help caregivers recognize and manage care recipients' problem behaviors and subsequently monitor levels of caregiver mastery before depressive symptoms become apparent. In particular, clinicians should monitor caregivers with high level of depressive symptoms before or at the onset of the care situation.

Investigators in other areas of caregiving have reported that caregiver mastery might be improved by implementing educational and cognitive behavioral interventions (Gitlin et al., 2003), and health care providers in neuro-oncology might be able to apply similar interventions to ultimately decrease caregivers' depressive symptoms. Increasing caregiver knowledge and comfort in the care situation can lead to higher levels of caregiver mastery (Mahoney, 2003), which is particularly vital to caregivers of people with PMBTs; these caregivers have reported needing information and assistance in dealing with problem behaviors (Sherwood et al., 2004). Clinicians might improve caregiver mastery by helping caregivers integrate education on problem behaviors and neuropsychiatric sequelae, teaching caregivers better ways to cope with problems (such as realizing the aberrant behavior is a result of the tumor, rather than intentional behavior of the care recipient), and providing stress-reducing techniques such as relaxation and guided imagery.

The second component of the partial mediation model showed that a portion of the relationship between care recipients' problem behaviors and caregivers' depressive symptoms was unaffected by caregiver mastery. These findings indicate that care recipients' problem behaviors can directly lead to caregivers' depressive symptoms, a relationship that has not been well explored in caregiving literature related to oncology. Research with caregivers of people with dementia has shown that care recipients' problem behaviors are one of the strongest and most consistent predictors of caregiver distress (Pinquart \& Sörensen, 2004). Meta-analyses have shown that when both behavioral and functional problems are present in care recipients, behavioral problems are so difficult to deal with that limitations in care recipients' physical function do not lead to emotional distress over and above that caused by problem behaviors (Pinquart \& Sörensen, 2004). Consistent findings indicate to clinicians the importance of monitoring problem behaviors in care recipients as a predictor of caregivers' depressive symptoms and the need to intervene with caregivers when problem behaviors become more frequent. In particular, clinicians should pay particular attention to the risk for depressive symptoms in younger caregivers and female caregivers.

The differential role of caregiver mastery in caregiver outcomes also has implications for future research. Findings have shown the importance of caregiver mastery as a predictor or mediating variable in oncology caregiver research and the need to understand other personal caregiver characteristics that might change the way in which predictors of caregiver outcomes (such as problem behaviors) affect caregivers' depressive symptoms.

Data from this study provide a beginning look at the way in which nonsociodemographic caregiver characteristics influence the relationship between care recipients' problem behaviors and caregiver depressive symptoms. A crosssectional design was useful in beginning to explore these relationships, but it does not allow for inferences regarding how caregiver mastery influences relationships over time. Second, the sample consisted primarily of Caucasian female spouses of people with PMBTs. Although the situations in this sample were consistent with how this disease exists in the general population, the design and sample do not allow for generalizability, particularly to various ethnic groups or to men. Third, care recipients' behavior problems were rated by proxy by the caregiver. People who have lower levels of caregiver mastery or higher levels of depressive symptoms might have reported care recipients as more impaired than would an objective assessor (Bookwala \& Schulz, 1998). Finally, the number of months that family members had been providing care varied widely (although more than 3/4 of the sample had been providing care for less than 3 years). Although differences in time allowed for variations in levels of caregiver mastery, it may also have reflected other differences in caregivers which were not measured or identified in this study, ultimately limiting generalizability.

\section{Conclusions}

This study was a beginning step in examining the relationship between caregiver mastery and care recipients' behavior problems in outcomes for caregivers in oncology. Study results were consistent with the work of Nijboer et al. (2000) and Gaugler et al. (2005) who describe the role of caregiver mastery in oncology caregivers and extend the science of caregiving research by more closely examining how 
caregiver mastery functions in the presence of care recipients' behavior problems. Data from studies such as these are vital in an era of cost containment in health care amidst concerns about the clinical applicability and cost effectiveness of interventions. Because behavior problems in care recipients place some family caregivers at risk for negative outcomes, further determining the role of caregiver mastery can help to ensure more efficient and effective delivery of caregiver interventions in oncology.

\section{References}

Andresen, E., Malmgren, J., Carter, W., \& Patrick, D. (1994). Screening for depression in well older adults: Evaluation of a short form of the CES-D. American Journal of Preventive Medicine, 10, 77-84.

Baron, R., \& Kenny, D. (1986). The moderator-mediator variable distinction in social psychological research: Conceptual, strategic and statistical considerations. Journal of Personality and Social Psychology, 51, 11731182.

Bennett, J. (2000). Focus on research methods. Mediator and moderator variables in nursing research: Conceptual and statistical differences. Research in Nursing \& Health, 23, 415-420.

Bookwala, J., \& Schulz, R. (1998). The role of neuroticism and mastery in spouse caregivers' assessment of and response to a contextual stressor. Journals of Gerontology Series B-Psychological Sciences \& Social Sciences, 53(3), 155-164.

Bookwala, J., \& Schulz, R. (2000). A comparison of primary stressors, secondary stressors, and depressive symptoms between elderly caregiving husbands and wives: The caregiver health effects study. Psychology and Aging, 15, 607-616.

Gaugler, J., Hanne, N., Linder, J., Given, C., Tolbert, V., Kataria, R., et al. (2005). Cancer caregiving and subjective stress: A multi-style, multidimensional analysis. Psycho-Oncology, 14, 293-302.

Gitlin, L.N., Belle, S., Burgio, L., Czaja, S., Mahoney, D.F., GallagherThompson, D., et al. (2003). Effect of multicomponent interventions on caregiver burden and depression: The reach multisite initiative at 6month follow-up. Psychology and Aging, 18, 361-374.

Kaufer, D.I., Cummings, J.L., Ketchel, P., Smith, V., MacMillan, A., Shelley, T., et al. (2000). Validation of the npi-q, a brief clinical form of the neuropsychiatric inventory. Journal of Neuropsychiatry \& Clinical Neurosciences, 12, 233-239.

Kenny, D. (2005). Mediation. In Encyclopedia of statistics in behavioral science (pp. 1194-1198). New York: John Wiley and Sons.

Kiecolt-Glaser, J., Preacher, K., MacCallum, R., Atkinson, C., Malarkey, W., \& Glaser, R. (2003). Chronic stress and age-related increases in the proinflammatory cytokine il-6. Proceedings of the $\mathrm{Na}$ tional Academy of Sciences of the United States of America, 100, 9090-9095.
Kozachik, S.L., Given, C.W., Given, B.A., Pierce, S.J., Azzouz, F., Rawl, S.M., et al. (2001). Improving depressive symptoms among caregivers of patients with cancer: Results of a randomized clinical trial. Oncology Nursing Forum, 28, 1149-1157.

Li, L.W., Seltzer, M.M., \& Greenberg, J.S. (1999). Change in depressive symptoms among daughter caregivers: An 18-month longitudinal study. Psychology \& Aging, 14, 206-219.

Mahoney, D.F. (2003). Effects of an automated telephone support system on caregiver burden and anxiety: Finding from the REACH intervention study. Gerontologist, 43, 556-567.

Mahoney, R., Regan, C., Katona, C., \& Livingston, G. (2005). Anxiety and depression in family caregivers of people with Alzheimer's disease: The laser-ad study. American Journal of Geriatric Psychiatry, 13, 795801.

Nijboer, C., Tempelaar, R., Triemstra, M., Van Den Bos, G.A., \& Sanderman, R. (2001). The role of social and psychologic resources in caregiving of cancer patients. Cancer, 91, 1029-1039.

Nijboer, C., Triemstra, M., Tempelaar, R., Mulder, M., Sanderman, R., \& Van Den Bos, G. (2000). Patterns of caregiver experiences among partners of cancer patients. The Gerontologist, 40, 738-746.

Northouse, L.L., Mood, D., Templin, T., Mellon, S., \& George, T. (2000). Couples' patterns of adjustment to colon cancer. Social Science \& Medicine, 50, 271-284.

O'Hara, R., Mumenthaler, M., Davies, H., Cassidy, E., Buffum, M., Namburi, S., et al. (2002). Cognitive status and behavioral problems in older hospitalized patients. Annals of General Hospital Psychiatry, 27(1), 1.

Ory, M., Hoffman, R., Yee, J., Tennstedt, S., \& Schulz, R. (1999). Prevalence and impact of caregiving: A detailed comparison between dementia and nondementia caregivers. The Gerontologist, 39, 177-185.

Pearlin, L., \& Schooler, C. (1978). The structure of coping. Journal of Health and Social Behavior, 19, 2-21.

Pinquart, M., \& Sörensen, S. (2004). Associations of caregiver stressors and uplifts with subjective well-being and depressive mood: A meta-analytic comparison. Aging \& Mental Health, 8, 438-449.

Radloff, L. (1977). The CES-D scale: A self-report depression scale for research in the general population. Applied Psychological Measurement, $1,385-401$.

Salander, P. (1996). Brain tumor as a threat to life and personality: The spouse's perspective. Journal of Psychosocial Oncology, 14, 1-18.

Sherwood, P., Given, B., Doorenbos, A., \& Given, C. (2004). Forgotten voices: Lessons from bereaved caregivers of persons with a brain tumor. International Journal of Palliative Nursing, 10, 67-75.

Sherwood, P., Given, B., Given, C., Schiffman, R., Murman, D., Lovely, M., et al. (2006). Predictors of distress in caregivers of persons with a primary malignant brain tumor. Research in Nursing and Health, 29(2), 105-120.

Skaff, M., Pearlin, L., \& Mullan, J. (1996). Transitions in the caregiving career: Effects on sense mastery. Psychology and Aging, 11, 247-257.

Sorensen, S., Pinquart, M., \& Duberstein, P. (2002). How effective are interventions with caregivers? An updated meta-analysis. Gerontologist, $42(3), 356-372$. 